\title{
SURFACE OXIDE FILM ENTRAINMENT MECHANISMS IN SHAPE CASTING RUNNING SYSTEMS
}

\author{
C Reilly $^{1}$, N.R Green ${ }^{2}$ and M.R. Jolly ${ }^{1}$ \\ ${ }^{1}$ School of Mechanical Engineering, University of Birmingham, UK \\ ${ }^{2}$ School of Metallurgy and Materials, University of Birmingham, UK
}

Keywords: Casting, Oxide, Quality Assessment, Entrainment, Running System, Hydraulic Jump

\begin{abstract}
During the transient phase of filling a casting running system surface turbulence can cause the entrainment of oxide films into the bulk liquid. Research has shown that these are detrimental to the material's integrity. Common mechanisms for this entrainment include returning waves, arising during filling of the runner bar, and plunging jets, found when pouring into a basin. One of these, the returning wave, has been studied in greater depth, using real-time X-ray and process modelling techniques alongside the application of physical principals. It has been concluded that when developed, returning waves cannot attain the more stable and less entraining tranquil flow regime desirable in the running system of castings.
\end{abstract}

\section{Introduction}

Real time X-ray studies have shown that when a stream of fluid impacts the end of a runner bar a chaotic flow regime is produced for a short period in this locality. This chaotic regime develops into a returning wave. These waves are known to be highly entraining and detrimental to casting integrity as they entrain double oxide films $[1,2]$. Such flow phenomena have previously been observed to resemble hydraulic jumps [3].

It was the original intention of this research to determine the threshold flow conditions that lead to formation of, and gas entrainment by, hydraulic jumps in liquid metals. A threshold has been shown experimentally in water [4-7], where the hydraulic jump is relatively well understood and the theory for which is reliant on certain assumptions being made [8, 9]. One of these; the assumption that surface tension effects are negligible may not hold true however for most liquid metals. After detailed experimental investigations, contrary to what had been shown through simulation previously [10], it was not possible to create hydraulic jumps within an open channel running system at liquid metal velocities in excess of $3 \mathrm{~ms}^{-1}$, flow depths of $0.01 \mathrm{~m}$ and flow distances of $1 \mathrm{~m}$. Thus it was concluded a 'trigger' is required to initiate a 'hydraulic jump' type structure. It was observed that in this geometric configuration only the return wave generated at the end of the runner created such a structure.

Returning waves have been known as entraining hydraulic structures but little work has been undertaken thus far on characterising them. Using a combination of experimental, numerical and first principles methods this work has concentrated on attempting to understand the critical features of a horizontal liquid stream within an open channel that results in the initiation of entrainment, in order to allow further insight into the constrained return wave found in running systems. 
The foundry engineer can calculate the returning wave velocity for a constrained wave in a runner bar of uniform thickness via the principles of conservation of volume [8], as represented in equation 1 . The difficulty comes in predicting the height of the returning wave (and thus wave velocity) when the constraint is removed.

Past work has shown how casting integrity is greatest when the system fills in a tranquil manner. However, if return waves are formed the persistence of these is critical in determining their overall damage to casting integrity $[11,12]$. Although the use of low profile runners, i.e. a height of less than the sessile drop height of the fluid, has been advocated to stop such waves forming [13] this is not always possible; for example due to manufacturing constraints or lack of flow control for multiple gated systems.

\section{Criterion Development}

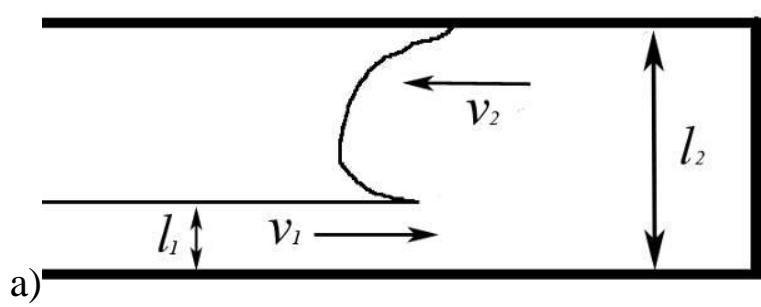

Figure.1 a) Constrained flow schematic

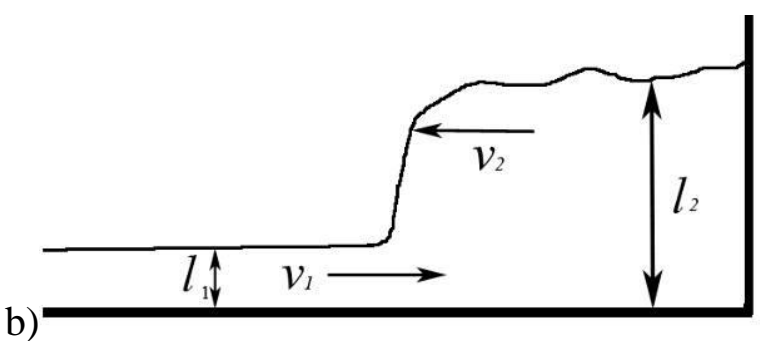

b)Unconstrained flow schematic

\section{Constrained Flow}

For a constrained return wave the velocity can be calculated from principles of conservation of volume [8] as shown in Equation 1 where $v_{1}$ is the inflowing fluid velocity $\left(\mathrm{ms}^{-1}\right), l_{1}$ is the inflowing fluid height (m), and $l_{2}$ is the runner bar height (m). This is shown in Figure 1(a).

$$
v_{2}=\frac{l_{1} \cdot v_{1}}{l_{2}-l_{1}}
$$

However, this condition, is only true for the section of the running system between the end of the runner and the in-gate (Section A, Figure 2) To calculate the return wave velocity for the area between the in-gate and downsprue (Section B, Figure 2) it is necessary to account for the fluid volume which is flowing into the casting volume.

For most industrial castings this is extremely difficult to determine from first principles without making assumptions that severely limit the accuracy of the calculation. One difficulty comes from the fact that the volume flowing into the casting varies with time.

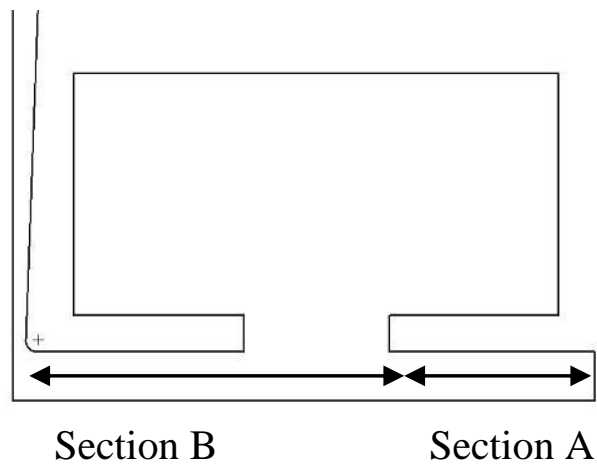

Figure 2. Casting schematic

\section{Unconstrained Flow}

For an unconstrained returning wave the velocity for a channel of uniform and unit thickness can be calculated by balancing the energy equation, as shown in the derivation below: (Figure 1b) 
$\begin{aligned} & \text { Incoming flow } \\ & \text { kinetic energy }\end{aligned}+\begin{aligned} & \text { Potential energy of } \\ & \text { fluid height } l_{1}\end{aligned}=\begin{aligned} & \text { Returning wave } \\ & \text { kinetic energy }\end{aligned}+\begin{aligned} & \text { Potential energy } \\ & \text { of fluid height } l_{2}\end{aligned}$

The potential energy (PE) of the incoming flow per unit width per unit time is equal to $\frac{m g h}{t}$ however time is taken to be $1 \mathrm{~s}$, therefore the potential energy is equal to $m g h$. Where $m$ is the mass, $g$ the acceleration due to gravity, $t$ is time and $h$ is the average fluid depth i.e half $l_{l}$.

$$
m=\rho l_{1} v_{1} \quad \text { and } \quad h=\frac{l_{1}}{2}, \text { therefore } \mathrm{PE}=\frac{1}{2} \rho l_{1}^{2} v_{1} g
$$

where $\rho$ is the fluid density $\left(\mathrm{kgm}^{-3}\right)$. The kinetic energy (KE) of the incoming flow per unit width per unit time $=\frac{1}{2} m v_{1}^{2}$

$$
\text { As in (2) } m=\rho v_{1} l_{1} \text { and } \mathrm{KE}=\frac{1}{2} \rho l_{1} v_{1}^{3}
$$

Potential energy of the return wave per unit width per unit time is equal to $\frac{m g h}{t}$ however time is taken to be $1 \mathrm{~s}$, therefore the potential energy is equal to $m g h$.

$$
m=\rho\left(l_{2}-l_{1}\right) v_{2} \text { and } h=\left(\frac{l_{2}-l_{1}}{2}+l_{1}\right) \text { therefore PE }=\frac{1}{2} \rho\left(l_{2}-l_{1}\right) v_{2}\left(\frac{l_{2}-l_{1}}{2}+l_{1}\right) g
$$

Kinetic energy of return wave per unit width per unit time $=\frac{1}{2} m v_{2}^{2}$; where wave depth $=l_{2}-l_{1}$,

$$
m=\rho\left(l_{2}-l_{1}\right) v_{2}, \text { therefore } \mathrm{KE}=\frac{1}{2} \rho\left(l_{2}-l_{1}\right) v_{2}^{3}
$$

Balancing the energies of incoming and returning flows (energy flux) gives;

$$
\begin{aligned}
\frac{1}{2} \rho g l_{1}^{2} v_{1}+\frac{1}{2} \rho l_{1} v_{1}^{3} & =\frac{1}{2} \rho\left(l_{2}-l_{1}\right) v_{2}\left(\frac{l_{2}-l_{1}}{2}+l_{1}\right) g+\frac{1}{2} \rho\left(l_{2}-l_{1}\right) v_{2}^{3} \\
g l_{1}^{2} v_{1}+l_{1} v_{1}^{3} & =\left(l_{2}-l_{1}\right) v_{2}\left(\frac{l_{2}-l_{1}}{2}+l_{1}\right) g+\left(l_{2}-l_{1}\right) v_{2}^{3}
\end{aligned}
$$

For this equation to be of use it needs to be solved independently of $v_{2}$ or $l_{2}$. Substituting $v_{2}$ from equation 1 and simplifying gives;

$$
g l_{1}^{2} v_{1}+l_{1} v_{1}^{3}=\left(l_{2}-l_{1}\right)\left(\frac{l_{1} \cdot v_{1}}{l_{2}-l_{1}}\right)\left(\frac{l_{2}-l_{1}}{2}+l_{1}\right) g+\left(l_{2}-l_{1}\right)\left(\frac{l_{1} \cdot v_{1}}{l_{2}-l_{1}}\right)^{3}
$$

Rearranging;

$$
0=\left(g l_{1}^{2} v_{1}+l_{1} v_{1}^{3}\right)-\left(l_{1} \cdot v_{1}\left(\frac{l_{2}-l_{1}}{2}+l_{1}\right) g+\left(l_{2}-l_{1}\right)\left(\frac{l_{1} \cdot v_{1}}{l_{2}-l_{1}}\right)^{3}\right)
$$

Equation 9 was solved in Matlab using the 'solve' function. The solutions are too long to detail here. Matlab was used to compute values from this solution by imputing values of $l_{1}$ and $v_{1}$.

Upon determining $l_{2}$ the velocity, $v_{2}$ of an unconstrained wave can be calculated by substitution into equation 1 giving the results plotted in Figures 3 and 4. 


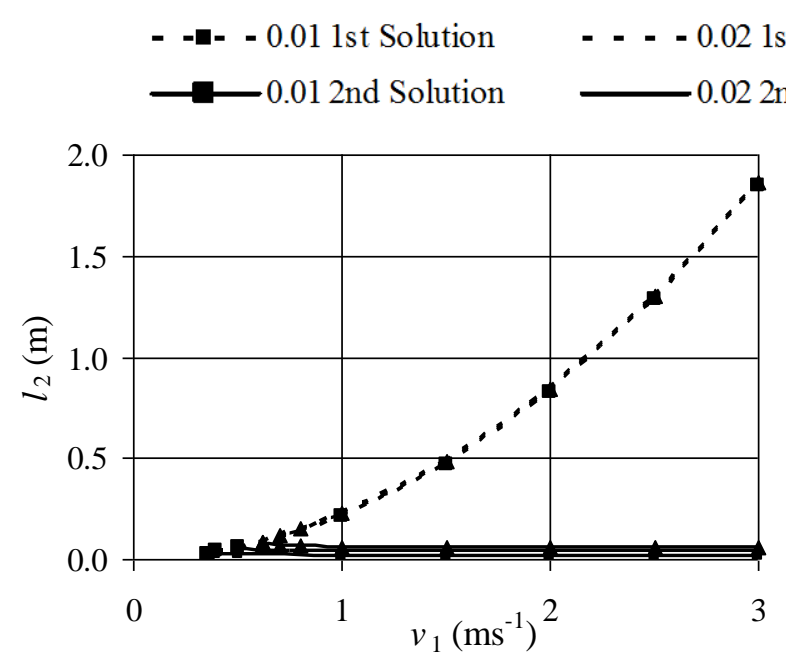

Figure 3. $v_{1} \mathrm{Vs}_{2}\left(l_{1}\right.$ of $0.01,0.02$ and $\left.0.03 \mathrm{~m}\right)$
- - - $\mathbf{-}-0.03$ 1st Solution

0.012 nd Solution

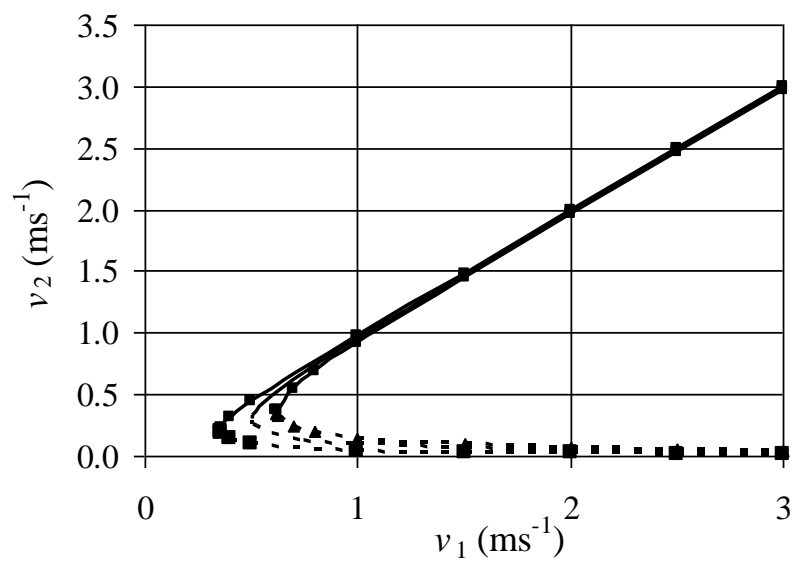

Figure 4. $v_{1} \mathrm{Vs}_{2}\left(l_{1}\right.$ of $0.01,0.02$ and $\left.0.03 \mathrm{~m}\right)$

\section{Experimental Design}

\section{Casting Experiments}

Moulds were cast in a real-time X-ray flow imaging facility, the principles of which have been reported previously [14]. Castings (Figure 5) were poured in resin bonded silica sand moulds (AFS grade 60 sand) using aluminium alloy A356. A range of sprue heights, whose design conforms to that defined by Campbell [1], were used to adjust the metal flow velocity and mass flow rate. The cast weight was $11 \mathrm{~kg}$, the pouring temperature $760{ }^{\circ} \mathrm{C}$ and the pouring was controlled using a robotic system. Filling was viewed using real-time $\mathrm{X}$-ray radiography to allow the qualitative assessment of the transient flow. Flow images were captured at a rate of $100 \mathrm{~s}^{-1}$ and resolution $800 \times 600$ pixels over a field of view of approximately $200 \times 150 \mathrm{~mm}$.

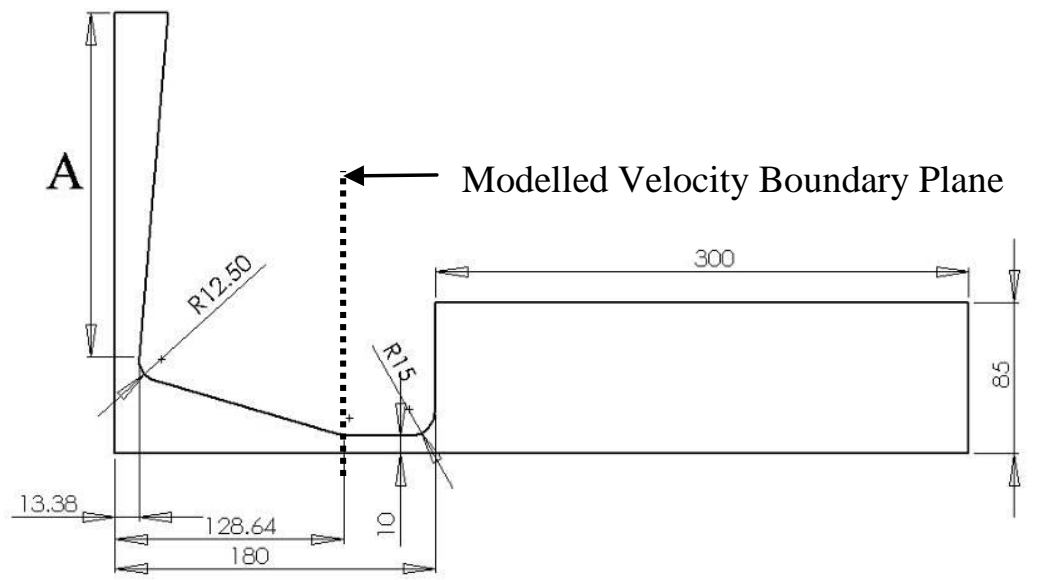

\begin{tabular}{|c|c|}
\hline Trial & A $(\mathrm{mm})$ \\
\hline 1 & 70 \\
\hline 2 & 105 \\
\hline 3 & 120 \\
\hline 4 & 200 \\
\hline 5 & 360 \\
\hline
\end{tabular}

Figure 5. Experimental mould (all dimensions in $\mathrm{mm}$ )

\section{Process Modelling}

Flow-3D [15], (a commercially available CFD code) was used to model the casting experiments. The software was run on PC's with dual core $2.6 \mathrm{GHz}$ processors and $8 \mathrm{~Gb}$ RAM. The mesh was 
$3 \mathrm{~mm}^{3}$ and contained 240,500 active cells. A velocity boundary condition was imposed on the plane shown in Figure 3. Velocities of 1.02, 1.1, 1.18, 1.25 and $1.37 \mathrm{~ms}^{-1}$ measured from the experimental results for $v_{1}$ from Trials 1 through 5 respectively were used as the boundary condition. Only the section of the mould to the right of the velocity boundary plane shown in Figure 3 was modelled. This allowed a more accurate comparison with the theoretical results as $v_{1}$ could be kept constant. However, it should be noted that with this approach the simulations may not fully match the real time X-ray results as during the transient filling phase when the downsprue is backfilling the pressure (and therefore velocity) of the fluid flowing through this section would not be expected to have reached the stable equilibrium as modelled. The RNG turbulence model and no slip wall boundary condition were applied. The material properties of, $2420 \mathrm{kgm}^{-3}, 0.00119 \mathrm{~Pa} \mathrm{~s}$ and $0.914 \mathrm{Nm}^{-1}$ were applied as density, viscosity and surface tension respectively [16].

\section{Results}

The results shown in Figures 3 and 4 show how, for a given set of inlet parameters, there are two alternative flow regimes; rapid and tranquil flow. The exception for this is where the energy is at a minimum at the 'nose' of the returning wave depth curve. This is analogous to the energy nose observed in hydraulic jump structures [8]. Rapid flow is defined as the condition when the flow takes the high velocity and low wave height parameter (second solution in the Figures). This regime is normally considered much more turbulent and highly entraining than the tranquil regime where the fluid takes the alternative parameters, namely a deep but slow moving flow that has a much reduced turbulence and no entrainment.

Figures 6 to 8 show examples of the experimental results. These show how initially the returning wave (circled in Figure 7) takes the shallow rapid flow form before immediately jumping to try and attain the tranquil depth.

Experimental results also show the returning wave periodically retreating along the runner bar, giving an insight of the potential magnitude of turbulent energy losses caused by the shearing in the region of the wave front. Figure $6 b, 0.2-0.4$ s shows clearly that the wave has progressed very little in 0.2 seconds when compared with the distance travelled between 0.4 and $0.6 \mathrm{~s}$.

Figure 7 shows two different, but clearly defined, examples of when the initial wave of low height jumps to attain a greater depth. This initial wave size matches the theoretical values calculated for Trials 1 through 5 , where $l_{1}$ equals $10 \mathrm{~mm} \pm 1 \mathrm{~mm}$ and $v_{1}$ ranges from $1.02 \mathrm{~ms}^{-1}$ to $1.35 \mathrm{~ms}^{-1}$. This gives $l_{2}$ a range of values between 18 to $22 \mathrm{~mm}$. This is obviously beyond the accuracy available with the real-time X-ray equipment where the uncertainty in flow depth is approximately of $\pm 1 \mathrm{~mm}$ at a turbulent free surface. However, this flow form can be seen in all experimental results, with the theoretical height of the initial wave lying within the experimental accuracy of the equipment. $l_{2}$ values of $18.1-22.2 \mathrm{~mm}$ at the wave nose was seen throughout the modelled results. However, with such transient waves being highly unstable, the accurate quantification of depth can be complicated. For example in Figure 6 (b) (0.6 s) the nose of the wave can be seen to be of uniform angle with $l_{2}$ values increasing from 15 to $35 \mathrm{~mm}$ with no clear definition of the expected $20 \mathrm{~mm}$ initial wave.

It can be seen in Figure 8 that despite the relatively low energy condition is entrainment of oxide films and bubbles, at the wave front. Further work is required to define if an entrainment 
threshold exists for a stable wave or whether the rapid regime is entraining for all inlet conditions.

With respect to the flow profiles observed the modelled flow results showed good agreement with the experimental data and first principles calculations. Figure 9 shows example images of the modelled returning wave form that initially advances in the rapid form before transition to the tranquil form. Figures 10 and 11 summarise experimental and Flow $3 D$ results which correlate favourably. However, correlation between these data and the calculated tranquil flow depths is at significant variance. In reviewing these results it must be remembered that;

i) The wave heights plotted in these Figures is not a measure of the initial wave height, but the height that the wave attains in trying to reach the tranquil flow regime.

ii) There are errors associated with extracting data from both the experimental results and modelled results. The error within the results is significant due to the following factors;

- The wave has not reached equilibrium, meaning results vary heavily depending on the time frame(s) chosen for analysis. Future work should look at using longer channels to allow a stable wave to form, giving more accurate analysis, although this would then reflect a departure from typical gating design.

- The bulking effect of air being entrained into the liquid changes the local fluid density; this effect it not considered in either the theoretical calculations or the model.

- The measuring of the incoming fluid velocity $v_{1}$ is subject to error as the initial jet is possibly unrepresentative of the steady state condition. It is well known that in the early stages of filling the effective head height is constantly varying as the sprue and basin back fill during this transient period.

- Difficulties exist in assessing experimental fluid depth; accuracy being $\pm 1 \mathrm{~mm}$.

- Parallax from the camera and further fluid surface topography contribute to an error up to $\pm 2 \mathrm{~mm}$.

It can be seen from equations 1 and 10 that the returning waves heights and velocities are independent of fluid density. For partial validation of this computer simulations were undertaken using Flow3D, these are shown in Figure 11. It was observed that as the density increased the surface profile also became more disturbed due to the strengthening momentum fields with in the flow Figure 11 (d)-(f). Further investigation is required to determine the extent of viscosity, density and surface tension on flow characteristics of fluid streams.

The computer model allows insight into the areas of greatest energy loss. Figure 12 shows how the area of greatest energy loss which is located at the shearing interface between the incoming flow and returning wave. It can also be seen that there is also noticeable energy dissipation by shear on the mould surface. 


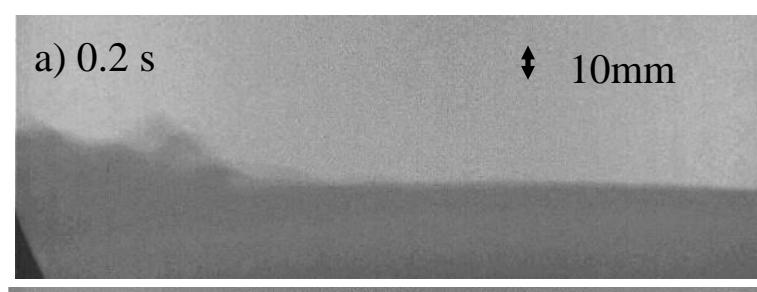

a) $0.4 \mathrm{~s}$

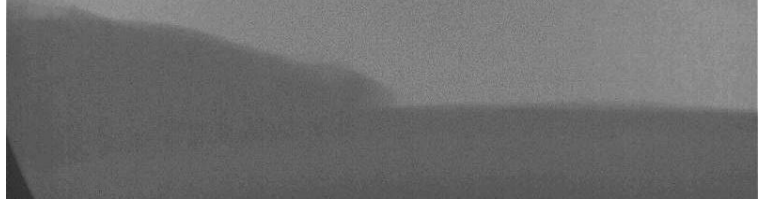

\section{a) $0.6 \mathrm{~s}$}
b) $0.2 \mathrm{~s}$

† $10 \mathrm{~mm}$ b) $0.4 \mathrm{~s}$

b) $0.6 \mathrm{~s}$

Figure 6. Real -time X-ray of returning waves in a)Trials1 and b) Trial 2
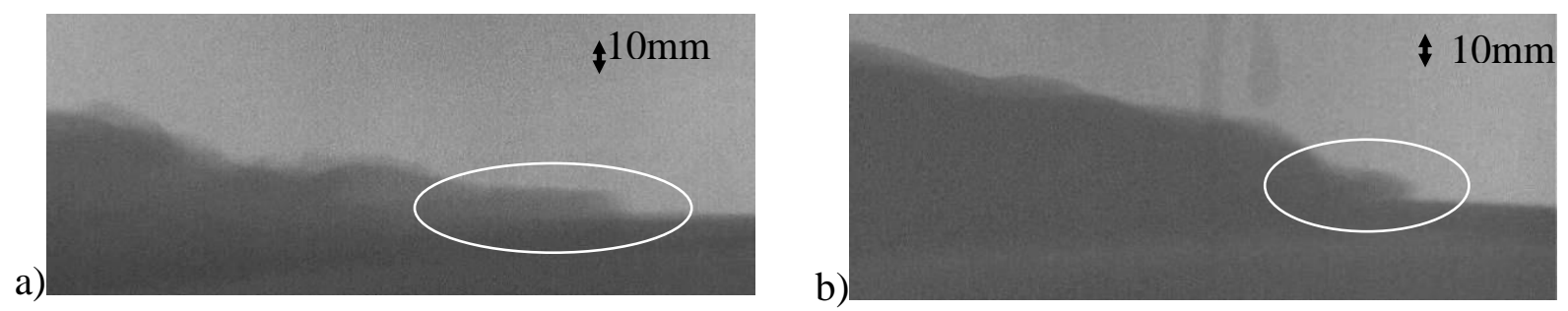

Figure 7. Images of initial wave height followed by a hydraulic jump. Ringed areas denote regions where the initial wave heights were measured.

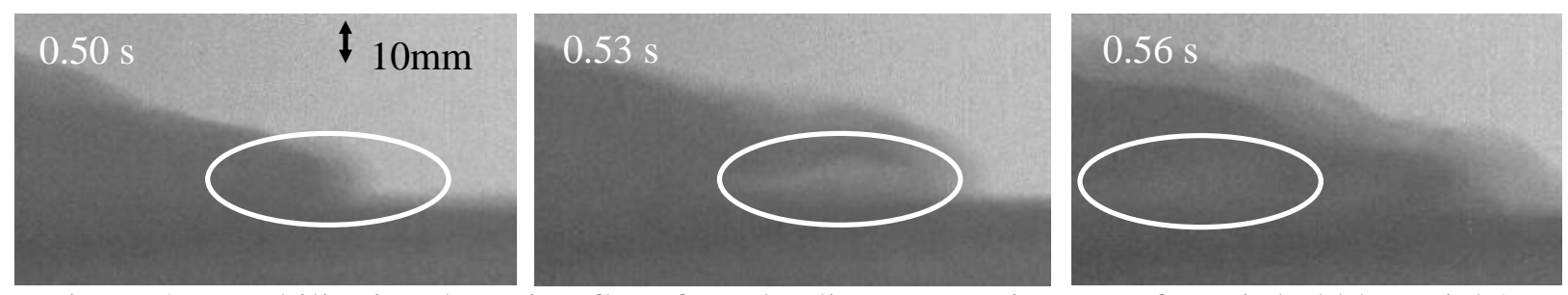

Figure 8. Instability in advancing flow front leading to entrainment of an air bubble; Trial 2

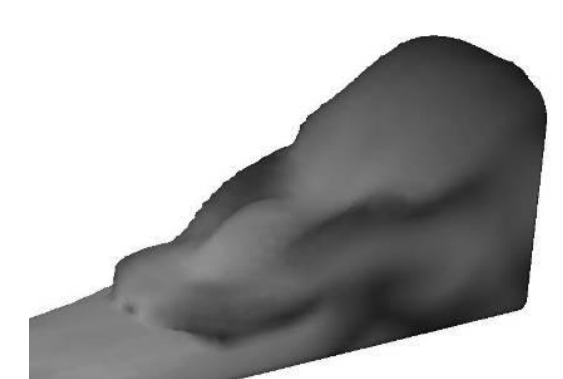

a) 3D Example of initial return wave followed by hydraulic jump (Trial 5)

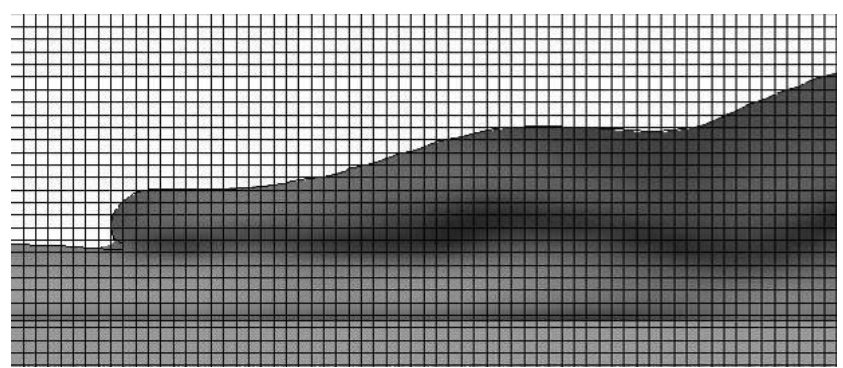

b) $2 \mathrm{D}$ section of returning wave, showing initial wave height followed by hydraulic jump.

(Mesh size $2 \mathrm{~mm}^{3}$ ) (Trial 2)

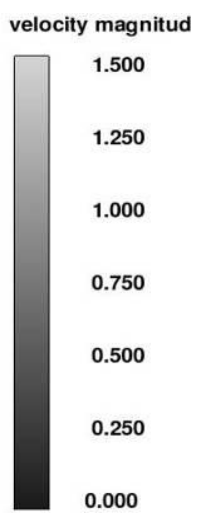

Figure 9. Examples of modelled two stage returning waves. 
- -₫ - Theoretical 1st Solution $\quad \cdot-\cdot$ Theoretical 2nd Solution $\longrightarrow$ Experimental Data $\rightarrow$ Flow 3D Data

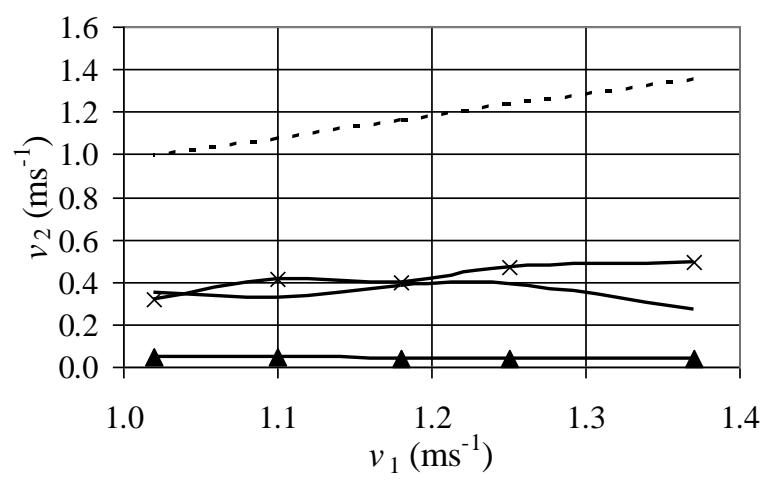

Figure 10. Plot of theoretical, modelled and measured return wave velocities.

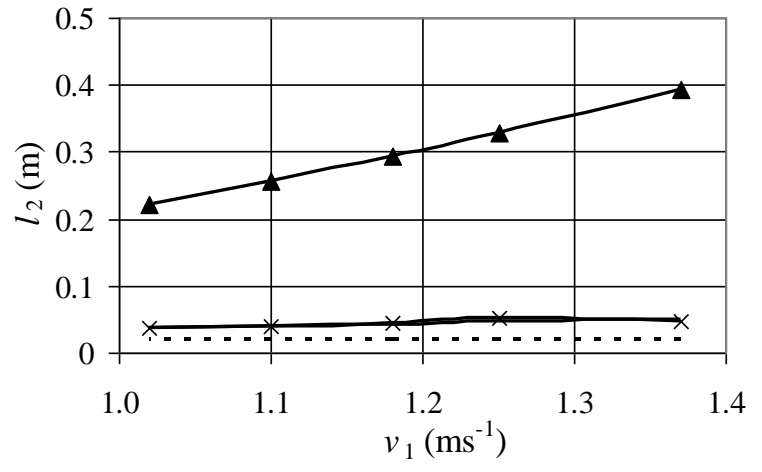

Figure 11. Plot of theoretical, modelled and measured return wave heights.

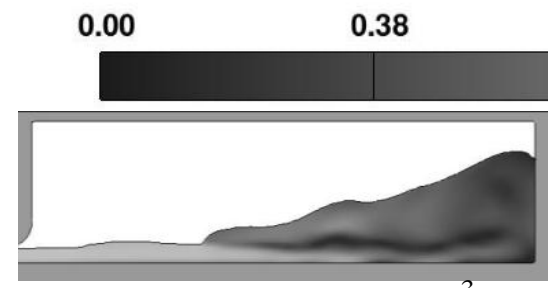

a) Density $2500 \mathrm{Kg} \mathrm{m}^{3}$ Time $=0.8 \mathrm{~s}$

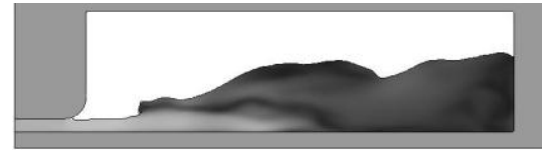

d) Density $2500 \mathrm{Kg} \mathrm{m}^{3}$

Time $=1.1 \mathrm{~s}$
0.75

1.50 Velocity Magnitude $\left(\mathrm{ms}^{-1}\right)$

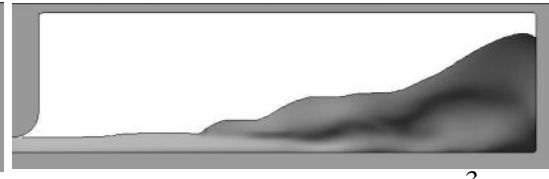

b) Density $5000 \mathrm{Kg} \mathrm{m}^{3}$ Time $=0.8 \mathrm{~s}$

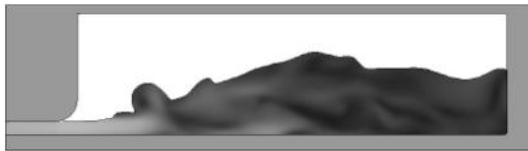

e) Density $5000 \mathrm{Kg} \mathrm{m}^{3}$

Time $=1.1 \mathrm{~s}$

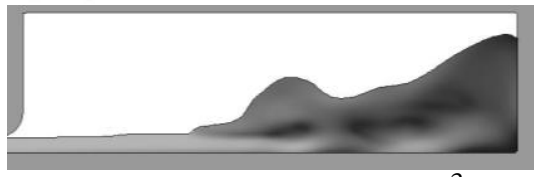

c) Density $7500 \mathrm{Kg} \mathrm{m}^{3}$ Time $=0.8 \mathrm{~s}$

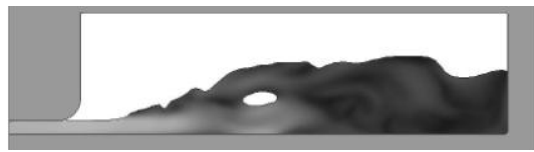

f) Density $7500 \mathrm{Kg} \mathrm{m}^{3}$ Time $=1.1 \mathrm{~s}$

Figure 11. Comparison of wave heights for three materials of different densities. Surface tension $\left(1 \mathrm{Nm}^{-1}\right)$ and viscosity $(0.001 \mathrm{~Pa} \mathrm{~s})$ remain the same. Fluid velocity is $1.18 \mathrm{~ms}^{-1}$

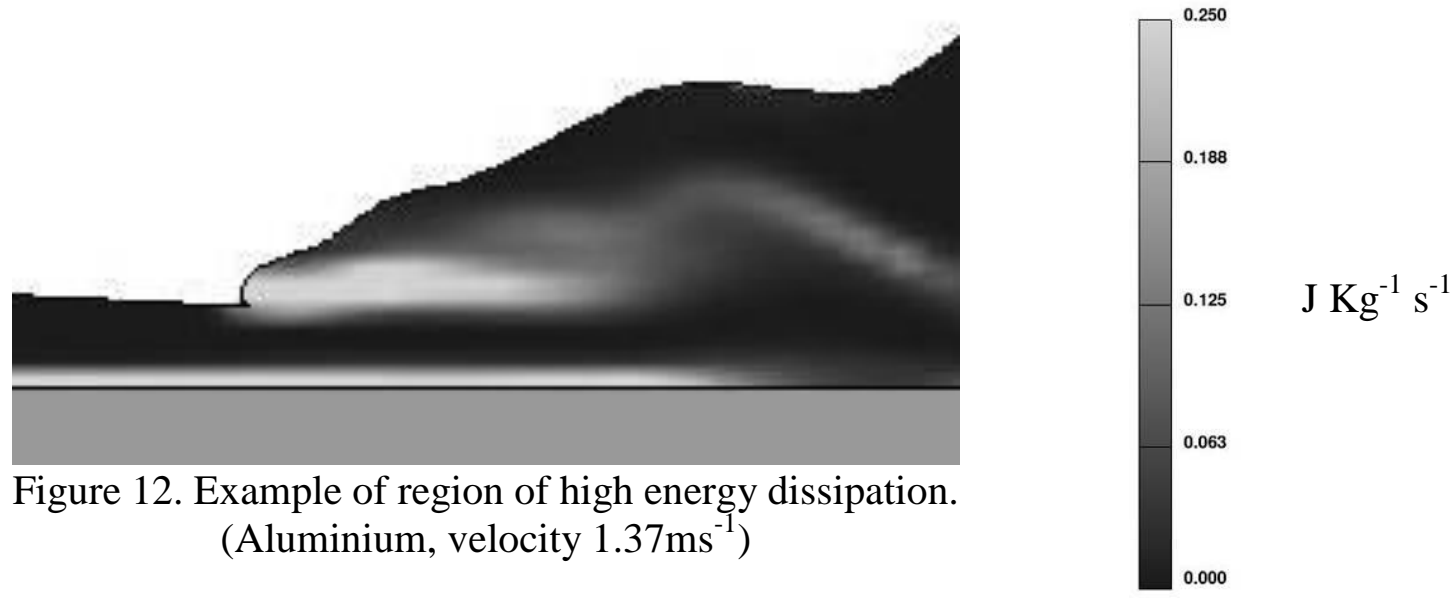

\section{Discussion}

It should be noted that the velocity $v_{2}$ and height $l_{2}$ of the returning wave for both constrained and unconstrained waves, are dependent on both the approach velocity $v_{1}$ and fluid depth $l_{1}$, but 
independent of the fluid density (Equation 8). The results seen in the modelling results of Figure 11 suggest this to be true although, at any given flow time, it was not unusual to observe substantial surface disturbances in any liquid density, but particularly at the highest density (Figure 11(f)). Similar flow instabilities were also observed in the real-time flow radiography (Figure 8) and reflects the highly turbulent nature of the shearing interface. However, it can also be seen that with increasing liquid density the surface becomes more disturbed. Since the other fluid parameters used in the model were constant this indicates some other fluid and flow properties that are not accounted for in the derivation of flow depths have a first order effect on the surface form. Since the founding work of Runyoro et al $[17,18]$ on critical in-gate velocities for liquid metals it is widely recognised that a liquid free surface becomes unstable (free surface turbulent) when surface tension forces are overcome by inertial pressure. In both the model the ratio of surface tension to density has not been accounted for.

In deriving the critical velocity at which a liquid becomes free surface turbulent, $v_{c r i t}$, Campbell obtained the expression [13]

$$
v_{c r i t}=\frac{2}{\sqrt{r}}\left(\frac{\gamma}{\rho}\right)^{1 / 2}
$$

in which $r$ is the radius of curvature of a hemispherical surface deformation, $\gamma$ the surface tension and $\rho$ the density. Thus for a fixed surface curvature the critical velocity for surface instability is reduced in proportion to $\left(\frac{\gamma}{\rho}\right)^{1 / 2}$. Therefore in the case of the liquid with density $7500 \mathrm{kgm}^{-3}$ the critical velocity for surface instability is reduced by a factor of 1.73 relative to that for the liquid with density $2500 \mathrm{kgm}^{-3}$ and this is considered the most likely explanation of the differences observed in the model.

In this study the well structured incoming flow had a Froude number in the range 3.3 to 4.4. In many casting systems even higher values could reasonably be expected. As stated in the introduction, the flow of liquid aluminium alloys under such inlet conditions has not been observed to initiate a hydraulic jump in a continuous open channel flow. The reflected wave and the shearing interface are essential components of the flow to initiate entrainment.

The presence of two stable flow depths suggested by the simple energy balance is analogous to that derived for an hydraulic jump [8,9]. In this series of experiments the greater (tranquil) calculated flow depth has not been observed and the predicted height remains somewhat surprising. It also appears to be the case that the thin jet forms ahead of the main returning flow and thus such systems will always be prone to severe entrainment. Therefore reduction of the persistence of the entrainment event is key to achieving optimum casting integrity [12]. One approach applied successfully in industry is the application of reticulated foam filters. When the fluid passes through the filter it is transformed to the tranquil flow depth, this allows most running systems to be primed in a single pass, therefore eliminating the return wave.

The energy losses within the system have been neglected within the derivation. This is because determination of energy loss coefficients is not possible without modelling or experimental work specific to each running system and its characteristics. These neglected energy losses include shear at the wave nose [19], shear on the mould walls [8] and internal bulk turbulence [7]. The viscosity of the fluid has been neglected in this study; this would have a significant effect on the magnitude of energy losses within the system as it is a measure of the fluids resistance to shear 
[8]. It can be seen in Figure 12 that turbulent shear losses are the main cause of energy dissipation. The viscosities for liquid metals commonly lie within half an order of magnitude of each other [20].

The returning flow depths observed experimentally suggest significant energy loss, due principally to shearing at the wave front, bulk turbulence and the bulking affect of air entrainment, Figure 12. It should also be noted that the tranquil depth of flow is far greater than that which could be accommodated in the runner bar of a casting design within normal parameters. Therefore, should the flow become constrained by the roof of a runner, causing the return wave to increase its velocity, high levels of entrainment will arise but low persistence. The turbulent energy loss can be quantified by calculating the energy difference between the flow obtained experimentally and that associated with either the rapid or tranquil flow regime using Equation 7. Calculation based upon the average return wave velocity and height derived from experimental data shows energy losses in the system of between 67 and $79 \%$. This is clearly well described within the RNG turbulence model used in Flow-3D.

All of the above findings show that unless a runner is a single pass design [1] there is no way to for it to fill without entrainment of oxide films. Single pass systems have been shown to be beneficial to casting integrity [21] but are traditionally not appropriate for systems with multiple gates or where large flow rates have to be used because of the casting section thickness. This is due to the fact that they have traditionally been designed so as to be less than the glissile drop height of the fluid. Previous experimental work at the University of Birmingham has shown that filters transform the incoming rapid flow to tranquil flow at the filter exit, Figure 13. Therefore, by incorporation of a filter close to the sprue exit a single pass system can be designed with flow within the runner in the tranquil, low velocity, regime. This deeper flow depth eliminates the drawbacks of using rapid flow single pass systems. Where single pass designs are not possible, it appears that running system geometry should concentrate on dissipation of the return wave energy [22]. Further research is required in this area.

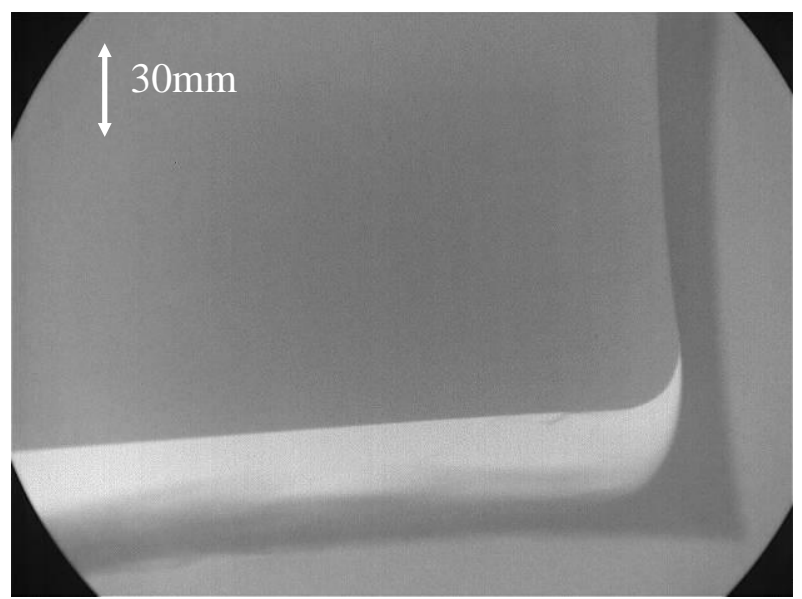

a) Rapid Flow

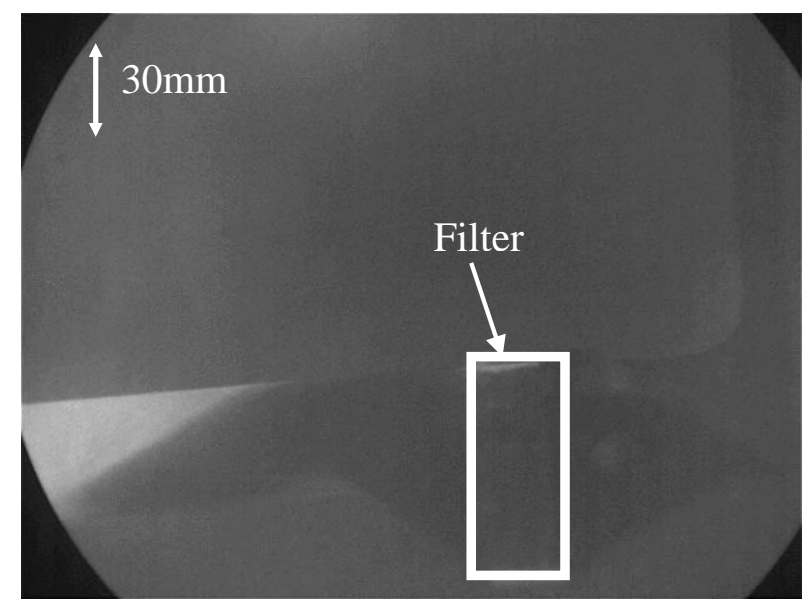

b) Tranquil flow regime initiated by filter

Figure 13. Transformation of flow regimes using a filter. Pour conditions and running systems identical. Filter 50 × 50 × 20 mm 20ppi reticulated foam filter 


\section{Conclusions}

1. Within the geometries studied it is impossible for the tranquil state to be achieved without an hydraulic jump and its inherent energy dissipation.

2. The trigger for an hydraulic jump to occur within a casting runner is the back wave.

3. There are two potential single pass systems, one in the rapid state and one in the tranquil state.

4. The use of a filter is required to initiate tranquil flow for a single pass system in the tranquil regime.

5. Returning back waves always develop an initial rapid regime before immediately trying to obtain stable tranquil state through an hydraulic jump.

6. Minimisation of the persistence of free surface entrainment is crucial to give maximum casting integrity.

7. Correlation between the theoretical model proposed for an unconstrained wave and experimental data is good. Further data are required for definitive validation

\section{Acknowledgments}

The authors would like to acknowledge the help of The School of Mechanical Engineering, The University of Birmingham for sponsoring the $\mathrm{PhD}$ of $\mathrm{CR}$, the support of Flow Science Inc and the EPSRC support of NG's chair (EP/D505569/1). 


\section{References}

[1] J. Campbell, Castings (Butterworth Heinemann, 1991).

[2] N. R. Green and J. Campbell, Influence in Oxide Film Filling Defects on the Strength of Al-7si-Mg Alloy Castings, Transactions of the American foundry society 114 (1994) 341 -347.

[3] B. Sirrell, M. Holliday and J. Campbell, The Benchmark Test 1995, Modelling of casting, welding and advanced solidification process VII (1995) 915-933.

[4] H. Chanson, Bubbly Flow Structure in Hydraulic Jump, European Journal of Mechanics, B/Fluids 26 (2007) 367-384.

[5] H. Chanson, Hydraulic Jumps: Bubbles and Bores, in "16th Australasian Fluid Mechanics Conference" (Crown Plaza, Gold Coast, Austraila, 2007).

[6] F. Murzyn and H. Chanson, Experimental Assessment of Scale Effects Affecting TwoPhase Flow Properties in Hydraulic Jumps, Experiments in Fluids 45 (2008) 513-521.

[7] H. Chanson, Air Bubble Entrainment in Free-Surface Turbulent Shear Flows (Academic press, London, 1996).

[8] B. S. Massey, Mechanics of Fluids 6th Edition (Chapman \& Hall, 1992).

[9] R. W. Fox and A. T. McDonald, Introduction to Fluid Mechanics (John Wiley and sons, 1985).

[10] F.-Y. Hsu, Further Developments of Running Systems for Aluminium Castings, (The University of Birmingham, 2003).

[11] C. Reilly, Surge Control Systems for Gravity Castings, in "The school of mechanical and manufacturing engineering" (The University of Birmingham, Birmingham, 2006).

[12] C. Reilly, N. R. Green, M. R. Jolly and J. C. Gebelin, Using the Calculated Fr Number for Quality Assessment of Casting Filling Methods, Modelling of casting, welding and advanced solidification process XII. (2009).

[13] J. Campbell, Castings 2nd Edition (Butterworth Heinemann, 2003).

[14] M. Cox, R. A. Harding and J. Campbell, Optomised Running System Design for Bottom Filled Aluminium Alloy 2199 Investment Castings, Materials science and technology 19 (2003) 613.

[15] Flow3D, Www.Flow3d.Com.

[16] K. C. Mills, Recommended Values of Thermophysical Properties for Selected Commercial Alloys (Woodhead publishing Ltd, 2002).

[17] J. Runyoro, S. M. A. Boutorabi and J. Campbell, Critical Gate Velocities for Film Forming Casting Alloys: A Basis for Specification, AFS Transactions 37 (1992) 225-234.

[18] J. J. Runyoro, Design of Gating Systems, in "School of Matallurgy and Materials" (The University of Birmingham, Birmingham, 1992).

[19] S. K. Misra, J. T. Kirby, M. Brocchini, F. Veron, M. Thomas and C. Kambhamettu, The Mean and Turbulent Flow Structure of a Weak Hydraulic Jump, Physics of Fluids 20 (2008) 035106.

[20] Metals Reference Book (Butterworths, London, 1976).

[21] M. Cox, R. A. Harding and J. Campbell, Optimised Running System Design for Bottom Filled Aluminium Alloy 2199 Investment Castings, Materials Science and Technology 19 (2003) 613-625.

[22] J. Campbell, Castings Practice: The Ten Rules of Casting (Butterworth Heinemann, 2004). 